\title{
Efficient photocatalytic degradation of organic dye from aqueous solutions over zinc oxide incorporated nanocellulose under visible light irradiation
}

https://doi.org/10.1515/mgmc-2020-0009

Received September 15, 2018; accepted April 29, 2020.

\begin{abstract}
Increased growth of textile industries leads to the tremendous accumulation of dyes on water and surrounding environments. This terrific increase of dyes is the major cause of water pollution which in turn adversely affects the aquatic lives and the balance of our ecosystem. Purpose of the present study is to report the synthesis and characterization of a composite namely zinc oxide incorporated nanocellulose $(\mathrm{ZnO} / \mathrm{NC})$ for effective degradation of an anionic dye, Congo red. Fourier transform infrared spectroscopy (FTIR), X-ray diffraction (XRD), Brunaeur, Emmett and Teller (BET) surface area analysis and scanning electron microscopy (SEM) studies have helped to characterize the composite. The optical properties of the samples were studied by UV-Visible spectroscopy. Feasibility of the photocatalyst in the degradation of Congo red was tested. Experimental conditions such as time of contact, concentration of the dye solution, catalyst dosage, $\mathrm{pH}$ were altered to find out the optimum conditions of degradation. The optimum $\mathrm{pH}$ was found to be 5.5 and dosage of $\mathrm{ZnO} / \mathrm{NC}$ was optimized as $0.075 \mathrm{~g}$ for a dye concentration of $20 \mathrm{ppm}$. Equilibrium was attained at $120 \mathrm{~min}$. The studies reveal that the photocatalyst $\mathrm{ZnO} / \mathrm{NC}$ is efficient for the photodegradation
\end{abstract}

\footnotetext{
* Corresponding author: Beena Bhaskaran, Department of Chemistry, Kumbalath Sankupilla Memorial Devaswom Board College, Sasthamcotta, Kollam, Kerala, India

Nahi Jamal, Department of Chemistry, Kumbalath Sankupilla Memorial Devaswom Board College, Sasthamcotta, Kollam, Kerala, India

Asha Radhakrishnan, Department of Chemistry, Devaswom Board Pampa College, Parumala, Kerala, India

Raveendran Raghavan, Department of Physics, Sree Narayana College, Kollam, Kerala, India
}

of Congo red. Photodegradation was due to electron hole interaction between metal oxides and nanocellulose.

Keywords: zinc oxide; sugar cane bagasse; photodegradation; photocatalyst

\section{Introduction}

In recent decades, due to rapid industrialization, huge amount of toxic effluents are being discharged to water bodies (Ansari et al., 2015). A large number of industries such as leather, paper, plastic, textile, food processing, printing, cosmetics and pharmaceuticals, etc., deal with dyes. The dye containing waste water can adversely affect the aquatic environment due to decreased sunlight penetration. In addition to these, some of the dyes can cause serious health problems such as mutation and cancer (Kumar et al., 2013). Serious issues of this water pollution are the shortage of safe water for drinking, household uses, agriculture, farming, etc., (Radhakrishnan et al., 2015). Therefore it is necessary to eliminate water pollution (Pourmortazavi et al., 2017). Physical methods such as adsorption and chemical methods such as chlorination and ozonation are some of the most frequently used methods for the removal of textile dyes (Chennakesavulu et al., 2015). Floculation and reverse osmosis are also some other methods commonly used, but these methods are not destructive. They just transfer the contaminants from one phase to another for which further treatments are required (Guillard et al., 2003). But Advanced oxidation process (AOP) like heterogeneous photocatalysis is a promising technology for water purification going on the surface of photocatalyst under the irradiation of photons, leading to total mineralization of dyes (Nagaveni et al., 2004), pesticides, organicinorganic pollutants and other deleterious pollutants. In 
photocatalysis, organic pollutants are degraded by using reusable photocatalysts, energetic light source such as sunlight or an artificial light and an oxidizing agent such as oxygen or air (Hachem et al., 2001). For a photocatalyst, as the particle size decreases, surface area increases and the number of active sites increases which leads to high reactivity (Alaton et al., 2002). As the number of active sites increases, the absorbance of pollutants on the surface of the catalyst increases (Augugliaro et al., 2002). The promoting of photoreaction in presence of a catalyst is termed as heterogeneous photocatalysis, a low cost and sustainable technology for the treatment of organic and inorganic pollutants present in water. In photocatalysis degradation takes place when the photocatalyst adsorbs photons of energy greater than its band gap energy (Anirudhan et al., 2015). A hole-electron generation occurs when the electron excites from the valence band to conduction band of the semiconductor. These electrons react with $\mathrm{O}_{2}$ to form $\mathrm{O}_{2}{ }^{-}$and holes react with $\mathrm{OH}^{-}$or $\mathrm{H}_{2} \mathrm{O}$ to form $\mathrm{OH}$ which are the active species to react with organic dyes.

Photocatalysis by semiconductors has gained much more attention due to its applications in hydrogen production, refractory pollutants elimination and selfcleaning surface. Wide band gap of metal oxides limit their ability to absorb visible light. Therefore many efforts such as metal or non-metal doping (Wang et al., 2014), composite synthesis (Mukherjee et al., 2014), etc., have been made to lower their band gap. Among these processes fabrication of composites with polymers has been shown to effectively lower the band gap and allows greater absorption in the visible region (Ansari et al., 2015). Such composites are expected to achieve synergetic and complementary behavior between the components.

In the present work we report the synthesis of a composite assembled from nano zincoxide $(\mathrm{ZnO})$ and nanocellulose (NC). Nano $\mathrm{ZnO}$ is a photocatalyst with excellent properties and has many applications. Semiconducting metal oxides have photocatalytic activities for the degradation of pollutants. Metal oxide nanoparticles like $\mathrm{ZnO}$ and $\mathrm{TiO}_{2}$ have been used to degrade non-biodegradable pollutants such as dyes via photocatalytic routes. In the present work, nanocellulose prepared from sugarcane bagasse was selected for the synthesis of composites with ZnO. Sugarcane bagasse is a waste product produced by the sugar and alcohol industries (Simkovic etal., 1990). It is used as a raw material for industrial applications like electricity generation, pulp and paper production and products based on fermentation (Pandey et al., 2000). Nanocellulose has been obtained from the inexpensive and readily available lignocellulosic material namely sugarcane bagasse by acid hydrolysis (Bondeson et al., 2006). For the preparation of NC, we hereby suggest a modified acid hydrolysis approach by an ecofriendly method. On the conversion of cellulose to nanocellulose, efficiency is enhanced due to the increase in the number of available functional groups, increase in surface area and crystalline nature. The NC prepared is made into a composite with nano $\mathrm{ZnO}$ and it is well characterized by different experimental techniques like XRD, FTIR, UV-Visible spectroscopy, SEM and BET surface area measurements. The photocatalytic activity of the materials was studied through the degradation of Congo red (CR). CR is an anionic dye frequently used in textile industry. Experiments were carried out to optimize the conditions like $\mathrm{pH}$, time, initial concentration and adsorbent dosage.

\section{Results and discussion}

\subsection{XRD analysis}

The XRD of the synthesized $\mathrm{ZnO}$ samples (Figure 1) show broad peaks at $2 \theta$ values of $31.9,34.6,36.4,47.7,56.7$, and 63.1 which are typical for the hexagonal $\mathrm{ZnO}$ wurtzite structure with lattice parameters a and c of 3.249 and $5.205 \mathrm{~A}^{\circ}$, respectively. The observed peaks of the nano crystallites match well with standard JCPDS files (36-1451), and no other crystalline phases were detected. The XRD of $\mathrm{NC}$ gives a broad hump between $16.1^{\circ}$ and $23.2^{\circ}$ indicating its amorphous nature. The XRD of $\mathrm{ZnO} / \mathrm{NC}$ confirms the formation of nanocomposites. The well-defined peaks

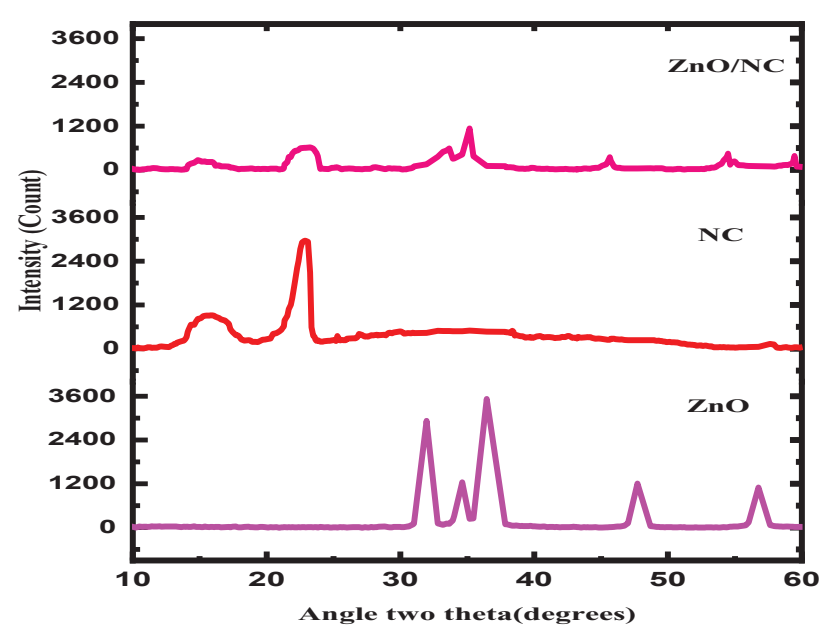

Figure 1: $\mathrm{XRD}$ of $\mathrm{ZnO}, \mathrm{NC}$, and $\mathrm{ZnO} / \mathrm{NC}$. 
corresponding to the crystal planes ( $\left.\begin{array}{lll}1 & 0 & 0\end{array}\right),\left(\begin{array}{lll}0 & 0 & 2\end{array}\right)$ and (1 0 1) of nano $\mathrm{ZnO}$ were found to be incorporated into the XRD of NC. It is thus clear that the metal oxide particles are well distributed in the nanocellulose matrix. Further, a change in intensity is observed in the characteristic peak of NC $\left(2 \theta=23.2^{\circ}\right)$ which again confirms the formation of nanocomposite. The average size of the particles was determined from full width at half maximum (FWHM) of the diffraction peaks using Scherrer's equation (Ahmmad et al., 2010):

$$
D=\frac{k \lambda}{\beta \cos \theta}
$$

where $K$ is the shape factor $(K=0.9), \beta$ represents the full width at half maximum (FWHM), $\theta$ is the Bragg angle and $\lambda$ is the wavelength of X-rays used. The particle size calculated for $\mathrm{ZnO}$ was found to be $26 \mathrm{~nm}$.

\subsection{FTIR analysis}

The FTIR spectrum of $\mathrm{ZnO}$ (Figure 2) shows bands at around $566 \mathrm{~cm}^{-1}$, which can be assigned to the vibrations of $\mathrm{Zn}$ (II)-O bonds. The absorption peaks observed at $\sim 2500 \mathrm{~cm}^{-1}$ and $\sim 1400 \mathrm{~cm}^{-1}$ may be due to the the adsorbed water and $\mathrm{CO}_{2}$ molecules. The characteristic peaks of cellulose (Li et al., 2012) at $3400 \mathrm{~cm}^{-1}, 2800 \mathrm{~cm}^{-1}$ (due to $\mathrm{O}-\mathrm{H}$ stretching and $\mathrm{C}-\mathrm{H}$ stretching vibrations), $1371 \mathrm{~cm}^{-1}$, and $897 \mathrm{~cm}^{-1}$ (due to $\mathrm{O}-\mathrm{H}$ bending and $\mathrm{C}-\mathrm{H}$ deformation vibration) are observed in the FTIR of NC prepared in the present study. In the FTIR spectrum of the composites, the main characteristic bands of $\mathrm{NC}$ and $\mathrm{ZnO}$ are retained. However, the incorporation of metal oxides leads to the shift of some bands of NC.



Figure 2: FTIR spectra of $\mathrm{ZnO}, \mathrm{NC}$, and $\mathrm{ZnO} / \mathrm{NC}$.

\subsection{SEM analysis}

The SEM results of the prepared samples are shown in Figure 3. SEM of ZnO consists of homogeneous; almost flower shaped nanostructures with less agglomeration. The SEM of NC reveals its sheet like morphology. ZnO/NC shows morphology entirely different from the flower like and sheet like morphologies of its components via $\mathrm{ZnO}$ and NC.

\subsection{UV analysis}

The room temperature UV-Visible spectra of the prepared samples were taken in the range of 250 to $600 \mathrm{~nm}$ are shown Figure 4. The spectrum of $\mathrm{ZnO}$ shows absorption peak at $380 \mathrm{~nm}$ which can be consigned to the intrinsic

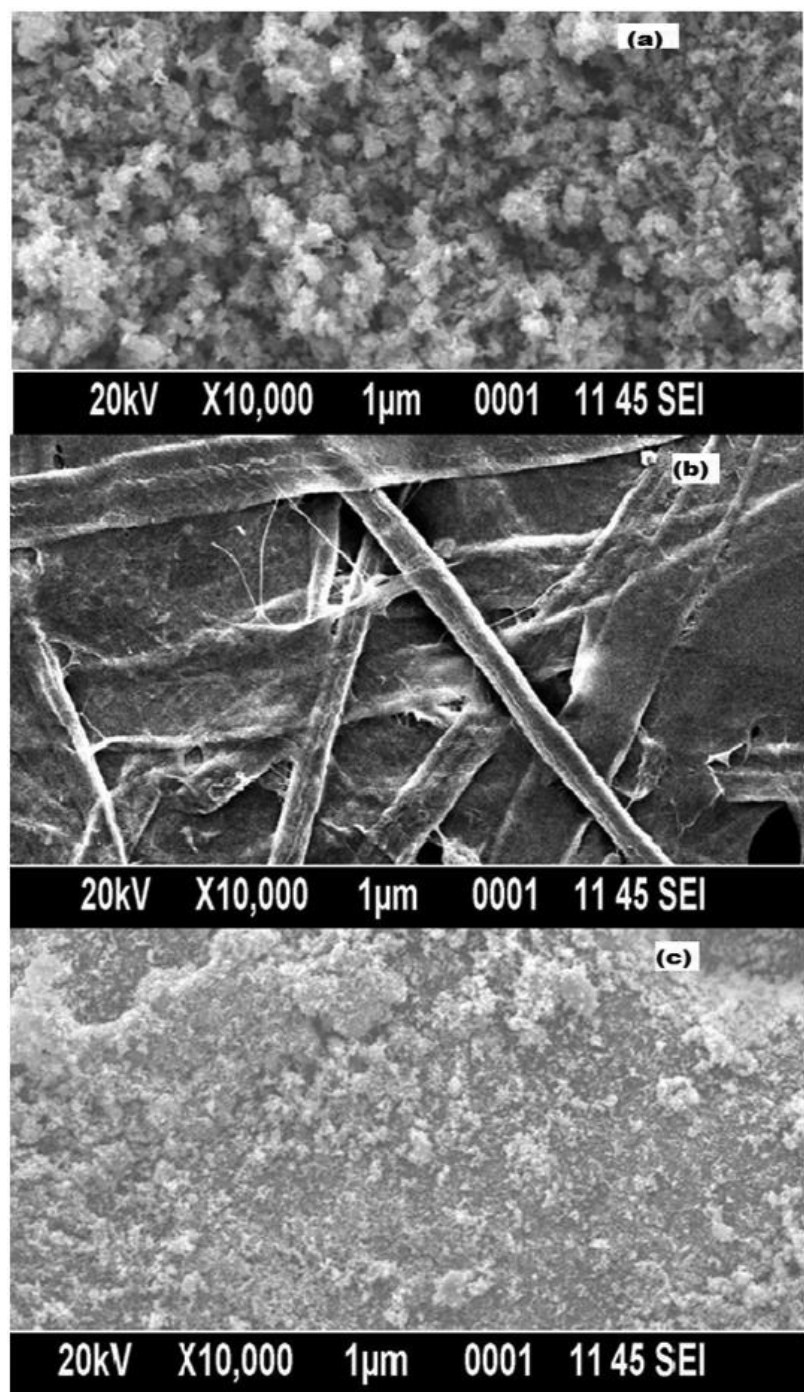

Figure 3: $\mathrm{SEM}$ images of (a) $\mathrm{ZnO}$, (b) $\mathrm{NC}$, and (c) $\mathrm{ZnO} / \mathrm{NC}$. 


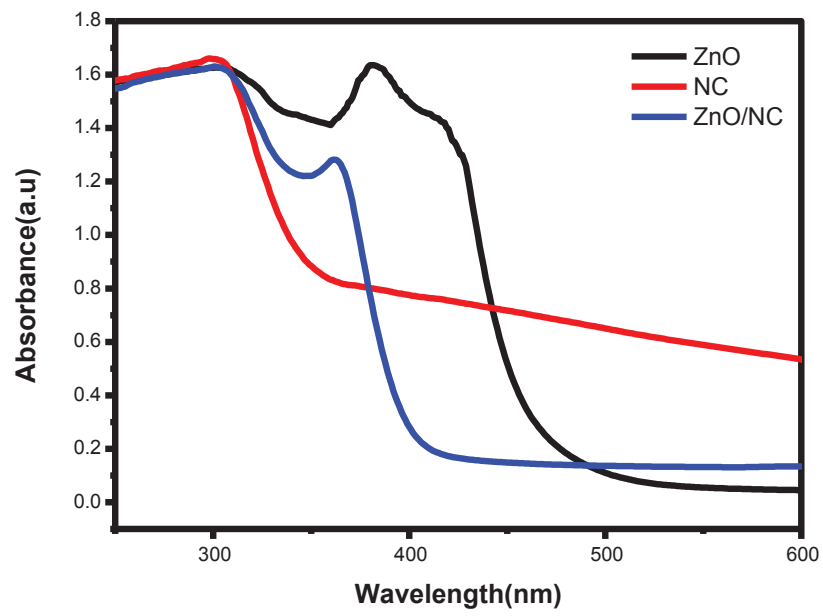

Figure 4: UV-Visible spectra of $\mathrm{ZnO}, \mathrm{NC}$, and $\mathrm{ZnO} / \mathrm{NC}$.

band gap absorption of $\mathrm{ZnO}$ due to the transition of electrons from valence band to conduction band $(02 p-$ Zn3d) (Khorsand Zak et al., 2011). The spectrum of NC shows absorption band with $\lambda_{\max }$ value of $300 \mathrm{~nm}$. When $\mathrm{ZnO}$ nanoparticles are doped into $\mathrm{NC}$, broad and strong absorption with a maximum at $360 \mathrm{~nm}$ was perceived, which was blue shifted relative to the $\mathrm{ZnO}(380 \mathrm{~nm})$ due to the quantum confinement (Prasad et al., 2006). The band gap of the samples was determined from the absorption spectra using Wood-Tauc's relation (Khorsand Zak et al., 2011).

$$
\alpha h \vartheta=D(h \vartheta-E g)^{n}
$$

where $h 9$ is the energy of the photon, $E g$ is the band gap of the material and $D$ is a constant. The band gap values obtained for $\mathrm{ZnO}, \mathrm{NC}$, and $\mathrm{ZnO} / \mathrm{NC}$ are 3.33, 3.8, and $2.75 \mathrm{eV}$, respectively. Thus the absorption of the composite is in the visible region due to its low band gap energy compared to that of $\mathrm{ZnO}$ and $\mathrm{NC}$.

\subsection{BET surface area measurement}

The Brunauer-Emmett-Teller (BET) surface area measured for $\mathrm{ZnO}, \mathrm{NC}$, and $\mathrm{ZnO} / \mathrm{NC}$ were found to be 82.3, 75.9, and $184.5 \mathrm{~m}^{2} / \mathrm{g}$, respectively. Surface area of the composite $\mathrm{ZnO} / \mathrm{NC}$ is highest which also accounts for its good photo catalytic activity.

\subsection{Evaluation of photocatalytic activity}

To study the efficiency of the composite for the degradation of dyes, a synthetic anionic dye namely Congo red was selected, the structures of which is shown in Scheme 1.

\subsubsection{Effect of time}

The changes in optical absorption spectra of Congo red using $0.1 \mathrm{~g}$ of photocatalyst for $25 \mathrm{ppm}$ of dye solution of $\mathrm{pH} 7.5$ irradiated under visible light irradiation for different time intervals are shown in Figure 5. From the plots it is evident that there is a decrease in intensity with increase in time for all the samples. High rate of photodegradation of the dye was observed with increase in time for all the samples. The photodegradation efficiency of NC for degradation of CR after a time interval of $120 \mathrm{~min}$ was found to be $52 \%$ whereas for $\mathrm{ZnO}$ it was $72 \%$ and for ZnO-NC 98\%.

\subsubsection{Effect of catalyst dosage}

As the catalyst dosage was increased from 0.025 to $0.25 \mathrm{~g}$ for $25 \mathrm{ppm}$ dye solution of $\mathrm{pH} 7.5$ and irradiated for $120 \mathrm{~min}$, the percentage degradation of the Congo red dye was found to increase for all the catalysts as shown in Figure 6. The increase in amount of catalyst load increased the number of active sites on the photocatalytic surface, which in turn, increased the number of hydroxyl radicals (Wang et al., 2007). It is also observed that further increase in the load of photocatalyst dosage decreased the degradation efficiency in all the samples. This is because, with increasing catalyst loading, though the number of active sites increases the penetration of light decreases due to shielding effect (Jang et al., 2006). In comparison with $\mathrm{ZnO}$ and $\mathrm{NC}$, very small amount of $\mathrm{ZnO} / \mathrm{NC}$ was required for maximum degradation of Congo red.

\subsubsection{Effect of concentration}

The effect of concentration of the dyes (Figure 7) on the degradation was observed by varying the initial concentration of dyes from 10 to $50 \mathrm{ppm}$ using $0.1 \mathrm{~g}$ of catalyst for $120 \mathrm{~min}$ of $\mathrm{pH} 7.5$ under visible light irradiation. At low concentrations, the rate of degradation and substrate concentrations are directly

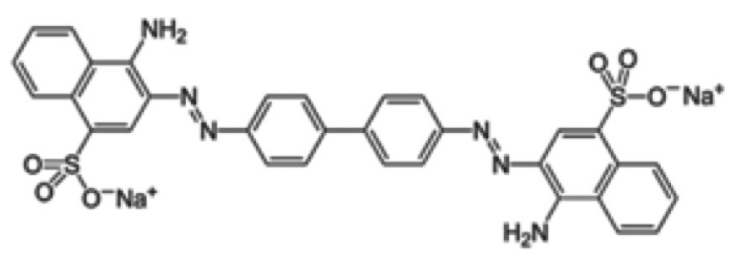

Scheme 1: Structure of Congo red. 

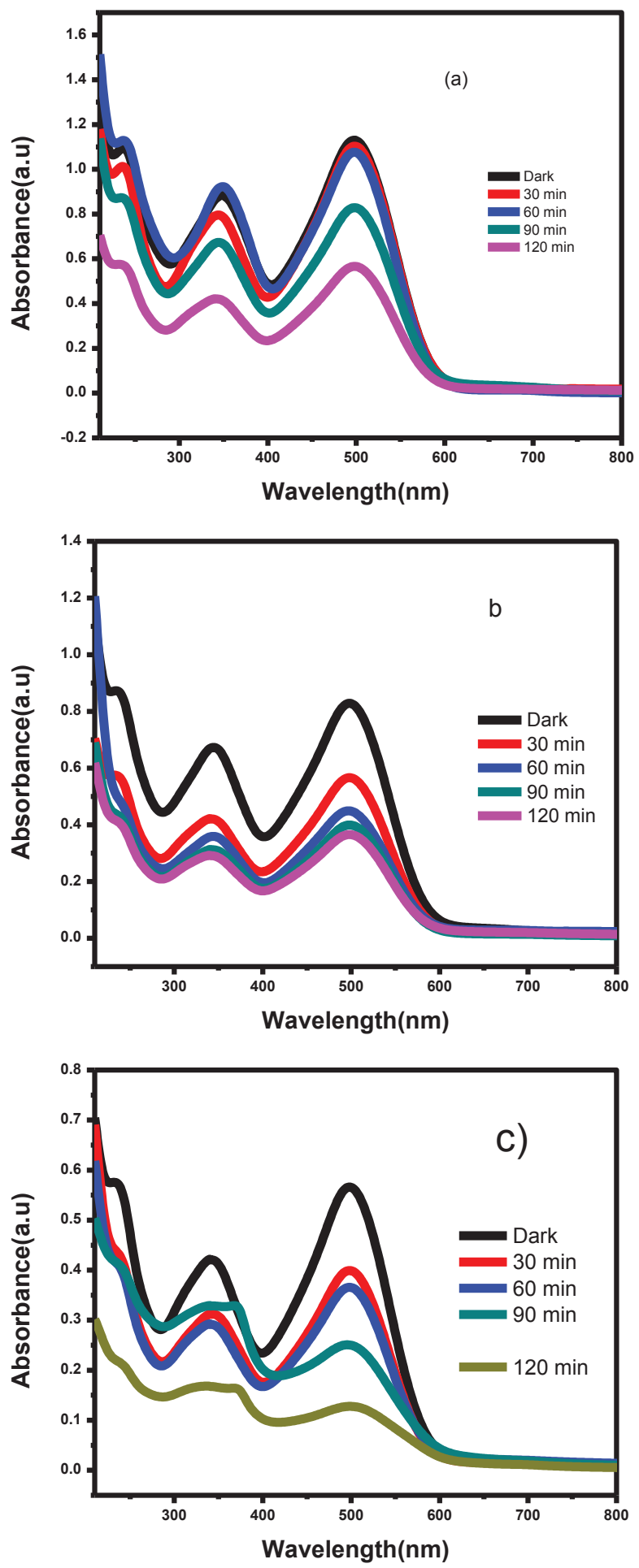

Figure 5: Time dependant absorption spectra of Congo red dye under visible light irradiation with (a) $\mathrm{ZnO}$, (b) $\mathrm{NC}$, and (c) $\mathrm{ZnO} / \mathrm{NC}$.

related. As the concentration increased, the degradation efficiency decreased for all the catalysts. The reduced rate of degradation at higher concentrations are due to unavailability of photoactive sites by increased adsorption of dye molecules, decreased rate of light penetration due

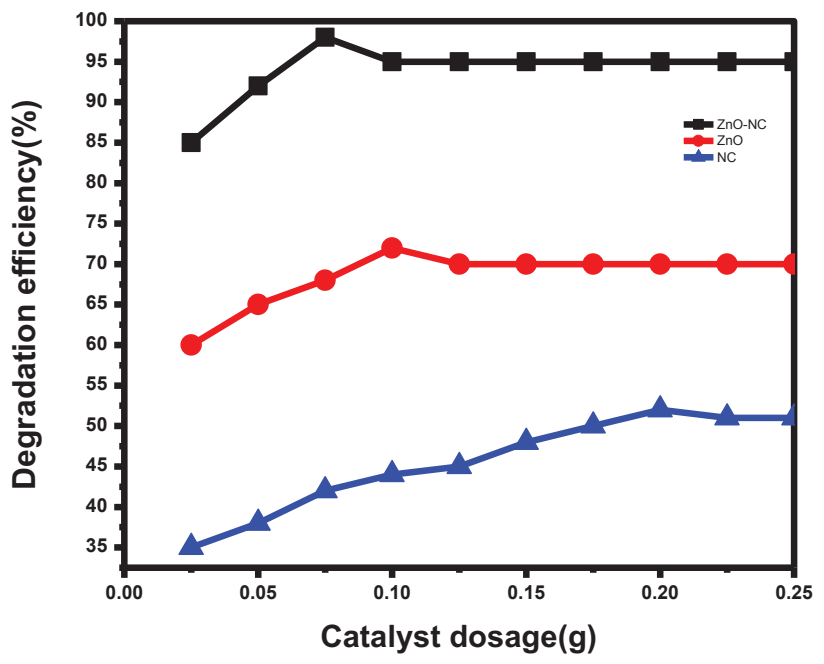

Figure 6: Degradation efficiency vs catalyst dosage for CR.

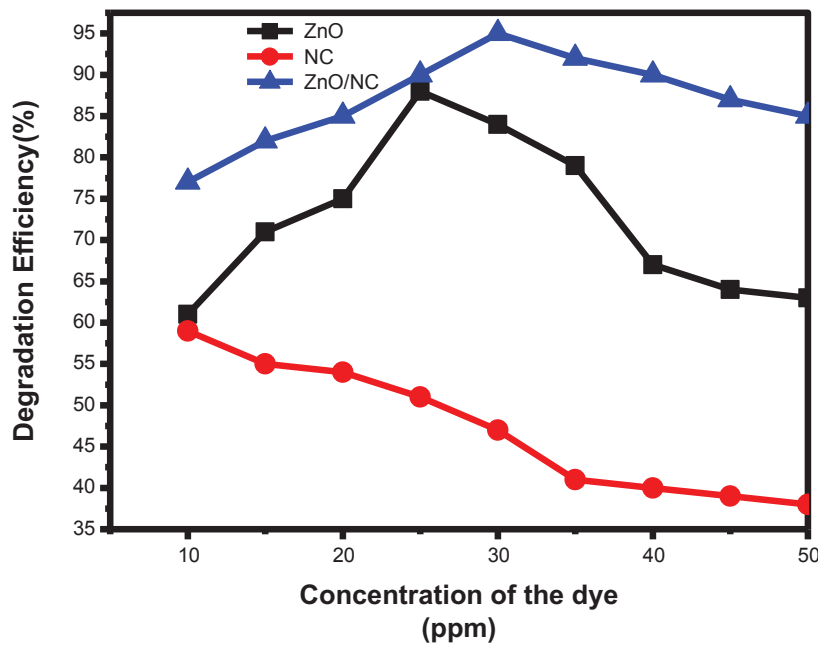

Figure 7: Degradation efficiency vs concentration for CR.

to screening effect and high rate of aggregation of dye molecules (Gopal et al., 2015). The highest degradation was obtained at concentrations 20, 25, and $30 \mathrm{ppm}$ respectively for $\mathrm{NC}, \mathrm{ZnO}$, and $\mathrm{ZnO} / \mathrm{NC}$.

\subsubsection{Effect of $\mathrm{pH}$}

Photodegradation capability of the photocatalyst depends on solution $\mathrm{pH}$. Studies were conducted to evaluate the effect of $\mathrm{pH}$ on the CR degradation using $25 \mathrm{ppm}$ dye solution and $0.1 \mathrm{~g}$ of photocatalyst for $120 \mathrm{~min}$ under visible light irradiation. For the degradation process, the effect of $\mathrm{pH}$ was studied over the $\mathrm{pH}$ range of 2.0-9.0 (Figure 8). The $\mathrm{pH}$ of the CR solution was altered by using diluted $\mathrm{HCl}$ or $\mathrm{NaOH}$. The results obtained are shown in Figure 9, which show a maximum degradation 


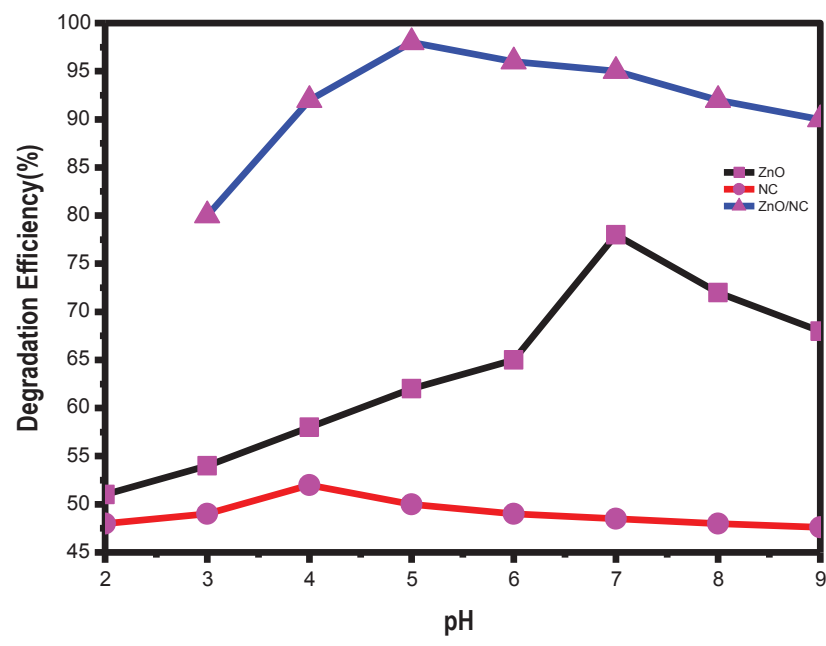

Figure 8: Degradation efficiency vs $\mathrm{pH}$ for $\mathrm{CR}$.

of $\mathrm{CR}$ at $\mathrm{pH} 7.5,4$, and 5.5 for $\mathrm{ZnO}, \mathrm{NC}$, and $\mathrm{ZnO} / \mathrm{NC}$ respectively. From literature it was found that the point of zero charge $\left(\mathrm{pH}_{\mathrm{pzc}}\right)$ for $\mathrm{ZnO}$ is 8 (Benhebal et al., 2013). So its surface charge is zero at this $\mathrm{pH}$. When the $\mathrm{pH}$ values are lower than $\mathrm{pH}_{\mathrm{pzz}}$, surface charge is positive and it is neutral at $\mathrm{pH}_{\mathrm{pzc}}$ and negative at higher $\mathrm{pH}$. As $\mathrm{CR}$ is an anionic dye, lower $\mathrm{pH}$ values show higher degradation efficiency.

Photodegradation of the dyes on the composites can be explained by the mechanism given in Figure 9. When metal oxide-nanocellulose composites are illuminated under visible light, the electrons are excited from the highest occupied molecular orbital (HOMO) to lowest unoccupied molecular orbital of (LUMO) NC and subsequently into the $\mathrm{CB}$ of metal oxide, holes will be left in the HOMO of NC. The electrons in the VB of metal oxide can migrate to the $\mathrm{HOMO}$ of $\mathrm{NC}$ and recombine with holes creating holes in the $\mathrm{VB}$ of metal oxide. As a result of this large number of electrons and holes are formed. These electrons react with $\mathrm{O}_{2}$ to form $\mathrm{O}_{2}{ }^{-}$and holes react with $\mathrm{OH}^{-}$or $\mathrm{H}_{2} \mathrm{O}$ to form $\mathrm{OH}$. which are the active species to react with organic dyes. The dyes undergo degradation through intermediates which are oxidized finally to $\mathrm{CO}_{2}$ and $\mathrm{H}_{2} \mathrm{O}$.

The mechanism of the degradation process can be expressed as

$$
\begin{aligned}
& \mathrm{MO}-\mathrm{NC} \rightarrow \mathrm{MO}-\mathrm{NC}^{+}+\mathrm{e}^{-} \\
& e^{-}+\mathrm{O}_{2} \rightarrow \mathrm{O}_{2}^{-} \\
& \mathrm{MO}-\mathrm{NC}^{+} \rightarrow \mathrm{MO}-\mathrm{NC}+\mathrm{h}^{+} \\
& \mathrm{OH}-+h^{+} \rightarrow \mathrm{OH}^{\cdot} \\
& \mathrm{H}_{2} \mathrm{O}+h^{+} \rightarrow \mathrm{OH}^{+}+\mathrm{H}^{+}
\end{aligned}
$$

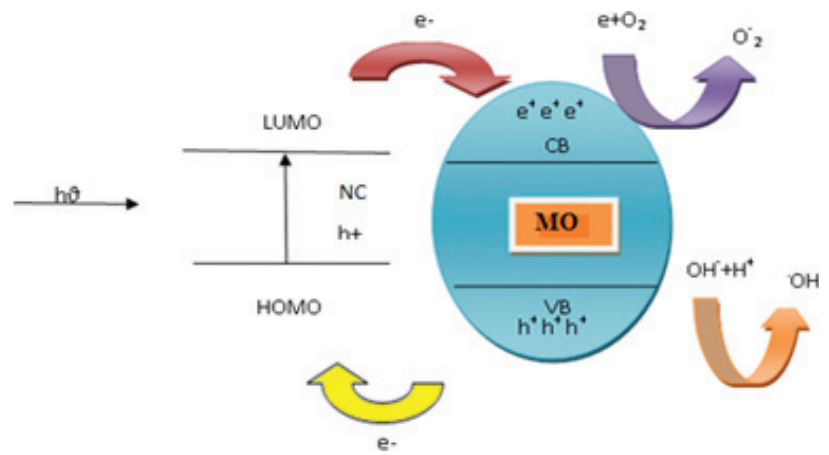

Figure 9: Mechanism of photodegradation by metal oxide nanocellulose composites.

$$
\text { Dye }+\mathrm{O}_{2}^{--} \text {or } \mathrm{OH} \rightarrow \text { intermediates } \rightarrow \mathrm{CO}_{2}+\mathrm{H}_{2} \mathrm{O}
$$

where $e^{-}$and $h^{+}$are the electrons and holes respectively.

Mostly semiconducting metal oxides are used as catalyst in the UV region. Here in the present study by the incorporation of $\mathrm{ZnO}$ into $\mathrm{NC}$ the resulting composite could be used as an effective photocatalyst in the visible region. Overall the process of photodegradation was found to be simple and complete. Almost complete (98\%) degradation was obtained using the nanocomposite. No significant degradation of the dye was observed when experiments were conducted without photocatalyst and without light. The composite prepared was from cellulose which is bio compactable, readily available and thus cost effective. The above study also points towards the utilization of such composites for the removal of pollutants from waterbodies.

\section{Conclusion}

The present work mainly covers the synthesis, characterization and photocatalytic degradation efficiency of $\mathrm{ZnO}, \mathrm{NC}$, and $\mathrm{ZnO}$ incorporated NC. Pure hexagonal phase $\mathrm{ZnO}$ with flower like morphology was synthesized using zinc acetate and HMT. Nanocellulose was prepared by a novel biogenic method using sugarcane bagasse. The composite of $\mathrm{NC}$ with $\mathrm{ZnO}$ was synthesized and well characterized. Photocatalytic degradation of the Congo red using the above materials was well studied. The study reveals that the catalytic activity of the composite is higher when compared to its counterparts. The variation in different experimental conditions such as time, concentration of the dye, catalyst dosage and $\mathrm{pH}$ that affects the photocatalytic degradation was also studied. 
The composite $\mathrm{ZnO} / \mathrm{NC}$ was found to be a promising catalyst for environmental applications.

\section{Experimental}

\section{Materials and methods}

All chemicals: zinc acetate dehydrate $\left(\mathrm{Zn}\left(\mathrm{CH}_{3} \mathrm{COOH}\right)_{2}\right.$ $\left.\mathrm{H}_{2} \mathrm{O}\right)$, hexa methylene tetraammine $\left\{\left(\mathrm{CH}_{2}\right)_{6} \mathrm{~N}_{4}\right\}$ (HMT), and Congo red (CR) were purchased from Merck Company as analytical grade and were used as received. Sugarcane bagasse was collected locally, cut into small pieces, washed and dried in sunlight. The dried bagasse was grained and the obtained powder was used for further studies.

\section{Preparation of $\mathrm{ZnO}$}

To an aqueous solution of $0.02 \mathrm{M}$ zinc acetate dihydrate (50 mL), a $0.25 \mathrm{M}$ hexa methylene tetraammine solution in water was added dropwise under constant stirring. The prepared solution was then stirred well for $120 \mathrm{~min}$ to obtain a homogeneously turbid liquid. This liquid was sonicated in an ultra-sonication bath for $30 \mathrm{~min}$. The curdy white precipitate obtained was cooled at room temperature, filtered, and washed with water followed by drying in hot air oven at $100^{\circ} \mathrm{C}$ for $2 \mathrm{~h}$. The main steps involved in the formation of $\mathrm{ZnO}$ are as follows (Rejani et al., 2014):

$$
\begin{aligned}
& \mathrm{CH}_{2} \mathrm{~N}_{4}+6 \mathrm{H}_{2} \mathrm{O} \rightarrow 4 \mathrm{NH}_{3}+6 \mathrm{HCHO} \\
& \mathrm{NH}_{3}+\mathrm{H}_{2} \mathrm{O} \rightarrow \mathrm{NH}_{4}^{+}+\mathrm{OH}- \\
& \mathrm{Zn}^{2+}+2 \mathrm{OH}-\rightarrow \mathrm{ZnO}+\mathrm{H}_{2} \mathrm{O}
\end{aligned}
$$

HMT a moderate alkali was used to generate hydroxide anion.

\section{Preparation of nanocellulose (NC) from sugar cane bagasse (SCB)}

The dried SCB was boiled with $25 \%$ (w/v) $\mathrm{NaOH}$ (fiber to liquid ratio 1:20) for $6 \mathrm{~h}$ and allowed to cool at room temperature. The obtained product was acid hydrolyzed with $50 \%(\mathrm{w} / \mathrm{v})$ sulfuric acid under stirring for another $6 \mathrm{~h}$. About $500 \mathrm{~mL}$ of distilled water was added to the above solution, cooled and centrifuged. This washing and centrifugation was continued for $24 \mathrm{~h}$. The obtained colloidal solution was then sonicated for $2 \mathrm{~h}$ in ice bath in order to avoid overheating (Dong et al., 1998). The suspension obtained was collected, dried, and stored for further studies.

\section{Preparation of $\mathrm{ZnO} / \mathrm{NC}$}

About $4 \mathrm{~g} \mathrm{NC}$ was dispersed in $50 \mathrm{~mL}$ water and stirred well for $1 \mathrm{~h}$. Simultaneously $2 \mathrm{~g} \mathrm{ZnO}$ was dispersed in $50 \mathrm{~mL}$ water and was stirred for $1 \mathrm{~h}$. The above solutions were mixed under constant stirring and sonicated for $3 \mathrm{~h}$ using ultra sonication bath. The obtained composite was washed and dried.

\section{Characterization}

X-ray diffractogram $\left(2 \theta=10-90^{\circ}\right)$ was obtained on XPERTPRO powder diffractometer with $\mathrm{Cu}-\mathrm{K} \alpha$ radiation. The FTIR spectrum was recorded using $\mathrm{KBr}$ wafer on the Thermo Nicolet FTIR model AVATAR 370 DTGS. In the present study, SEM of the sample was recorded using a JED-2300 system. The UV-Visible spectra were recorded at room temperature using JASCOV 650 UV-2550 UV Visible spectrophotometer. BET surface area of the samples was measured using SMART SORB 93 Surface area analyzer.

\section{Photocatalytic degradation of CR}

In a typical experiment, $0.1 \mathrm{~g}$ of the prepared catalyst was added to aqueous solution of CR (25 ppm, $300 \mathrm{~mL}, \mathrm{pH} 7.5)$ taken in a cylindrical photoreactor of capacity $500 \mathrm{~mL}$. The mixture was kept in the dark for 90 min to obtain equilibrium adsorption of CR onto the photo catalyst. The photoreactor was then illuminated with visible light (160 W, Hg vapour lamp) for $120 \mathrm{~min}$. Aliquant were withdrawn from the reaction mixture at regular time intervals and centrifuged immediately to remove the catalyst before absorbance was recorded using a JASCOV 650, UV-Visible spectrophotometer.

To study the effect of $\mathrm{pH}$, experiments were conducted in the $\mathrm{pH}$ range 2.0 to 9.0 using $0.1 \mathrm{~g}$ of photocatalyst with $25 \mathrm{ppm}$ of dye solution and 120 min of irradiation. To optimize the amount of photocatalyst, a series of experiments were carried out by varying the catalyst amount from 0.025 to $0.25 \mathrm{~g}$ for $25 \mathrm{ppm}$ dye solution of pH 7.5 irradiated for $120 \mathrm{~min}$. The effect of initial dye concentration was investigated by varying the initial concentration from 10 to $50 \mathrm{ppm}$ using $0.1 \mathrm{~g}$ of catalyst for $120 \mathrm{~min}$ of irradiation. Here also the $\mathrm{pH}$ of the solution was maintained at 7.5. Blank experiments were also performed in the absence of catalyst and absence of light. 
The photocatalytic degradation efficiency was calculated as follows (Radhakrishnan et al., 2016):

$$
\operatorname{Removal}(R \%)=\frac{C_{0}-C_{e}}{C_{0}} \times 100
$$

where $R \%$ is the degradation efficiency of CR, $C_{o}$ - initial concentration of dye solution $(\mathrm{mg} / \mathrm{L}), \mathrm{Ce}-$ equilibrium concentration of dye solution $(\mathrm{mg} / \mathrm{L})$.

Acknowledgements: The authors are thankful to the Management and the Principal of Kumbalath Sankupilla Memorial Devaswom Board College (K.S.M.D.B. College) for providing necessary laboratory facilities, STIC Cochin for instrumental analysis. One of the authors (N.J.) is thankful to the University of Kerala for financial assistance.

\section{References}

Ahmmad B., Kusumoto Y., Islam M.S., One-step and large scale synthesis of non-metal doped $\mathrm{TiO}_{2}$ submicrospheres and their photocatalytic activity. Adv. Powder Technol., 2010, 21, 292-297.

Alaton I.A., Balcioglu I.A., Bahnemann D.W., Advanced oxidation of a reactive dyebath effluent: comparison of $\mathrm{O}_{3}, \mathrm{H}_{2} \mathrm{O}_{2} / \mathrm{UV}-\mathrm{C}$ and $\mathrm{TiO}_{2}$ /UV-A processes. Water Res., 2002, 36, 1143-1154.

Anirudhan T.S., Deepa J.R., Binusreejayan, Synthesis and characterization of multi carboxyl-functionalized nanocellulose/ nanobentonite composite for the adsorption of uranium (VI) from aqueous solutions: Kinetic and equilibrium profiles. Chem. Eng. J., 2015, 273, 390-400.

Ansari M.O., Khan M.M., Ansari S.A., Cho M.H., Polythiophene nanocomposites for photodegradation applications: Past, present and future. J. Saudi Chem. Soc., 2015, 19, 494-504.

Augugliaro V. Baiocchi C., Bianco Prevot A., Garća-López E., Loddo V., Malato S., et al., Azo-dyes photocatalytic degradation in aqueous suspension of $\mathrm{TiO}_{2}$ under solar irradiation. Chemosphere, 2002, 49, 1223-1230.

Benhebal H., Chaib M., Salmon T., Geens J., Leonard A., Lambert S.D., Photocatalytic degradation of phenol and benzoic acid using zinc oxide powders prepared by the sol-gel process. Alexandria Eng. J., 2013, 52, 517-523.

Bondeson D., Mathew A., Oksman K., Optimization of the isolation of nanocrystals from microcrystalline cellulose by acid hydrolysis. Cellulose, 2006, 13, 171-180.

Chennakesavulu K., Madhusudhana Reddy M., Ramanjaneya Reddy G., Rabel A.M., Brijitta J., Vinita V., et al., Synthesis, Characterization and Photo catalytic studies of the composites by Tantalum oxide and Zinc oxide Nanorods. J. Mol. Struct., 2015, 1091, 49-56.

Dong X.M., Revol J.F., Gray D.G., Effect of Microcrystallite Preparation Conditions on the Formation of Colloid Crystals of Cellulose. Cellulose, 1998, 5, 19-32.

Gopal P., Mira P., Kim H.-Y., Lee S.-Y., Park S.-J., Electrospun ZnO hybrid nanofibers for photodegradation of wastewater containing organic dyes. A review. J. Ind. Eng. Chem., 2015, 21, 26-35.
Guillard C., Lachheb H., Houas A., Ksibi M., Elaloui E., Herrmann J.M., Influence of chemical structure of dyes of $\mathrm{pH}$ and of inorganic salts on their photocatalytic degradation $\mathrm{b} \mathrm{TiO}_{2}$ comparison of the efficiency of powder and supported $\mathrm{TiO}_{2}$. J. Photochem. Photobiol. A-Chem., 2003, 158, 27-36.

Hachem C., Bocquillon F., Zahraa O., Bouchy M., Decolourization of textile industry wastewater by the photocatalytic degradation process. Dyes Pigments, 2001, 49, 117-125.

Jang Y.J., Simer C., Ohm T., Comparison of zinc oxide nanoparticles and its nano-crystalline particles on the photocatalytic degradation of methylene blue. Mater. Res. Bull., 2006, 41, 67-77.

Khorsand Zak R., Razali R., Majid W.H.A., Darroudi M., Green synthesis and evolution of metabolic activity of starchmediated nanoceria. Int. J. Nanomed., 2011, 6, 1399-1403.

Kumar M., Sivakumar V., Tamilarasan R., Adsorption of Victoria blue by carbon/Ba/Alignate beads: Kinetics, Thermodynamics and Isotherm studies. Carbohydr. Polym., 2013, 98, 505-513.

Li J., Wei X., Wang Q., Chen J., Chang G., Kong L., et al., Homogeneous Isolation of Nanocellulose from Sugarcane Bagasse by High Pressure Homogenization. Carbohydr. Polym., 2012, 90, 1609-1613.

Mukherjee D., Barghi S., Ray A.K., Preparation and characterization of the $\mathrm{TiO}_{2}$ immobilized polymeric photocatalyst for degradation of aspirin under UV and solar light. Processes, 2014, 2, 12-23.

Nagaveni K., Sivalingam G., Hegde M.S., Madras G., Solar photocatalytic degradation of dyes high activity of combustion synthesized Nano-TiO . Appl. Catal. B-Env., 2004, 48, 83-93.

Pandey A., Soccol C.R., Nigam P., Soccol V.T., Biotechnological potential of agro-industrial residues I: Sugarcane bagasse. Bioresour. Technol., 2000, 74, 69-80.

Pourmortazavi S.M., Rahimi-Nasrabadi M., Aghazadeh M., Ganjali M.R., Karimi M.S., Norouzi P., Synthesis, characterization and photocatalytic activity of neodymium carbonate and neodymium oxide nanoparticles. J. Mol. Struct., 2017, 1150, 411-418.

Prasad V., Souza C.D., Yadav D., Shaikh A.J., Vigneshwaran N., Spectroscopic characterization of zinc oxide nanorods synthesized by solid-state reaction. Spectrochim. Acta A, 2006, 65, 173-178.

Radhakrishnan A., Rejani P., Bhaskaran B., CuO/polypyrrole nanocomposites as a marker of toxic lead ions for ecological remediation in contrast with $\mathrm{CuO}$ and polypyrrole. Main Group Met. Chem., 2015, 38, 133-143.

Radhakrishnan A., Rejani P., Shanavas Khan J., Bhaskaran B., Effect of annealing on the spectral and optical characteristics of nano ZnO: Evaluation of adsorption of toxic metal ions from industrial waste water. Ecotoxicol. Environ. Saf., 2016, 133, 457-465.

Rejani P., Radhakrishnan A., Bhaskaran B., Photo Catalytic Decomposition of Malachite Green in Aqueous Solutions under UV Irradition Using Nano ZnO Rod. Iranica J. Energy Environ., 2014, 5, 233-239.

Simkovic I., Mlynar J., Alfoldi J., Micko M.M., New aspects in cationization of lignocellulose materials. XI. Modification of bagasse with quaternary ammonium groups. Holzforschung, 1990, 44, 113-116.

Wang H., Xie C., Zhang W., Cai S., Yang Z., Gui Y, Comparison of dye degradation efficiency using $\mathrm{ZnO}$ powders with various size scales. J. Hazard. Mater., 2007, 141, 645-652.

Wang Y., Zhang R., Li J., Li L., Lin S., First-principles study on transition metaldoped anatase $\mathrm{TiO}_{2}$. Nanoscale Res. Lett., 2014, 9, 46-53. 